\title{
Language matters! Sprachliche Hindernisse für heterogene Lerngruppen in GW-Schulbüchern erkennen
}

\author{
*johanna.anich@stud.sbg.ac.at, Universität Salzburg \\ "*sandra.stieger@phsalzburg.at, Institut für Gesellschaftliches Lernen und Politische Bildung, PH Salzburg Stefan Zweig, ( $\triangle$ korresp. Autorin)
}

eingereicht am: 23.12.2020, akzeptiert am: 16.02.2021

\begin{abstract}
Die sprachliche Heterogenität von Lernenden steigt stetig. Folglich braucht es sprachsensible Zugänge in allen Unterrichtsfächern. Anhand einer exemplarischen, qualitativen und quantitativen strukturierenden Inhaltsanalyse von drei ausgewählten GW-Schulbüchern der Sekundarstufe I werden potenzielle sprachliche Herausforderungen auf Wort-, Satz-, Text- und visueller Ebene aufgezeigt. Das angewandte Analyseinstrument schärft den Blick für sprachliche Hindernisse und erleichtert die Einschätzung und Prüfung der Eignung von Unterrichtsmedien bzw. -materialien für heterogene Lerngruppen.
\end{abstract}

Keywords: Sprachliche Bildung, Sprachförderung, Geographie und Wirtschaftskunde Schulbuch, Inhaltsanalyse

\section{Language matters! Recognizing linguistic barriers for heterogeneous learning groups in geography and economics textbooks}

The linguistic heterogeneity of the students is constantly increasing. Consequently, language-sensitive approaches are needed in all subjects. Based on a qualitative and quantitative content analysis of three selected geography and economics textbooks of lower secondary education, potential language barriers on word, sentence, text, and visual level are exemplified. The applied analysis tool sharpens the view for language barriers and facilitates the assessment and testing of the suitability of instructional media or teaching materials for heterogeneous learning groups.

Keywords: language education, promotion of language learning, geography and economics textbook, content analysis

\section{$1 \quad$ Einleitung}

\section{„Schulbücher sind eine zentrale Lerngrund- lage in nahezu allen Fächern. Wissen wird in Schulbüchern primär über Sprache vermittelt“ (BMUKK 2012: 3).}

Die sprachliche Heterogenität in den Klassen nimmt zu. Das ist nichts Neues und auch immer wieder Gegenstand bildungspolitischer Diskurse (vgl. Vock \& Gronostaj 2017: 5). Vielfach wird hierbei die Bedeutung sprachsensiblen Unterrichtens oder sprachlicher Fördermaßnahmen im Zusammenhang mit Migration und dem wachsenden Anteil von Lernenden mit Deutsch als Zweitsprache argumentiert (vgl. Carnevale \& Wojnesitz 2014: 11). Allerdings haben auch vielfach Schüler*innen, die einsprachig aufgewachsen sind, Schwierigkeiten im Erwerb von (bildungs-)sprachlichen Kompetenzen. Besonders davon betroffen sind Kinder und Jugendliche aus bildungsfernen Schichten. Defizite im Bereich der Sprachkompetenz können nicht nur zu Verständnisproblemen beim Lesen von Texten und beim Bearbeiten von Aufgabenstellungen führen, sondern den Erwerb aller Kompetenzen beeinflussen (vgl. BMUKK 2012: 4).

Deshalb ist die spezifische Förderung sprachlicher Kompetenzen nicht nur Aufgabe des Sprachunterrichts, sondern ein Bildungsanliegen für alle Unterrichtsfächer. Dementsprechend weist jedes Unterrichtsfach in den Lehrplänen seinen Beitrag zu den Bildungsbereichen Sprache und Kommunikation aus (vgl. BMUKK 2012: 11) ${ }^{1}$. Hinzu kommt, dass jedes einzelne Unterrichtfach eine eigene Fachsprache hat,

\footnotetext{
Hinzukommt der im Jahr 2017 neu verordnete Grundsatzerlass Leseerziehung, der Anliegen der Sprachförderung mit Fokus auf Texterschließung stärken soll (vgl. BMBWF 2017).
} 
so auch das Unterrichtsfach GW ${ }^{2}$. Damit sind zusätzliche sprachliche Besonderheiten verbunden, die sich auf den unterschiedlichen Ebenen sprachlicher Anforderung äußern. Dazu zählen beispielsweise der Fachwortschatz auf der Wortebene, grammatische Strukturen auf der Satzebene und spezifische Textstrukturen auf der Textebene. Um diese sprachlichen Merkmale der Fachsprache zu erwerben, benötigen Schüler*innen Sprachbewusstsein und Übung (vgl. Schroeter-Brauss et al. 2018: 145).

Schulbücher, die ein wichtiges Unterrichtsmedium im (GW-)Unterricht sind (vgl. Hintermann et al. 2014: 81; vgl. Sitte 2011: 247 ff.), bedienen sich neben der Fachsprache auch der Bildungssprache. Im Gegensatz zur Alltagssprache sind Fach- und Bildungssprache von Schriftsprachlichkeit und abstrakten Inhalten geprägt (vgl. Carnevale \& Wojnesitz 2014: 8). Kennzeichen sind ihre Distanzierung, Komplexität und Objektivität. Der erfolgreiche Umgang mit der Bildungs- und Fachsprache kann über den Schulerfolg der Kinder und Jugendlichen entscheiden (vgl. BMUKK 2012: 4). Das In-Beziehung-Setzen von Bildungssprache und Alltagssprache ist damit eine zentrale Herausforderung für Lehrende und Lernende.

$\mathrm{Ob}$ und wie GW-Schulbücher den Forderungen einer sprachsensiblen Bildung entsprechen, wollen wir anhand einer exemplarischen qualitativen und quantitativen Inhaltsanalyse unter der Forschungsfrage Welche potenziellen sprachlichen Hindernisse lassen sich exemplarisch anhand eines Schulbuchkapitels identifizieren? aufzeigen. Für die Beantwortung der Forschungsfrage haben wir ein Analysetool entwickelt, das die Empfehlungen des BMUKK (2012) bezüglich der Sprachverwendung in Schulbüchern adressiert. Wir sind uns bewusst, dass eine produktorientierte Analyse keine Aussagen zum Unterrichtseinsatz von Schulbüchern zulässt. Dennoch sind wir überzeugt, dass die Erkenntnisse einen Beitrag zur Bewusstseinsbildung für sprachliche Hindernisse, die mit einem Einsatz eines Schulbuchs verbunden sein können, leisten. Entsprechend dieser Zielsetzungen erörtern wir im ersten Abschnitt aktuelle Forschungsergebnisse über die Bedeutung von Sprache im GW-Unterricht, um davon ausgehend im zweiten Abschnitt das Forschungsdesign unserer Studie vorzustellen. Nach der Darstellung der Ergebnisse der Studie (Abschnitt 4) und ihrer Diskussion (Abschnitt 5), fassen wir die daraus gewonnenen Erkenntnisse in einem abschließenden Fazit (Abschnitt 6) zusammen.

\footnotetext{
Im aktuellen Lehrplanentwurf für die Sekundarstufe I soll sprachliche Bildung durch sprachsensible Aufgabenstellungen und Lesestrategien Anwendung finden, um Lernende beim Erwerb der Fachsprache zu unterstützen (vgl. Lehrplankommission GW 2021: 2).
}

\section{Sprachbewusster GW-Unterricht?}

\author{
"Sprache ist das wichtigste Medium jeden \\ Unterrichts, auch des Geographieunterrichts" \\ (Budke \& Kuckuck 2017: 7).
}

Zur (fachlichen) Vermittlung von Informationen wird Sprache benötigt. Die Diskussionen über geographische und ökonomische Zusammenhänge erfolgen sprachlich, und Arbeitsergebnisse müssen sprachlich formuliert werden. Es zeigt sich also, Sprache ist die Grundlage aller Lehr- und Lernprozesse und das in jedem Unterrichtsfach. Allerdings ist die geographiedidaktische Forschung in diesem Bereich unzureichend. Wie Budke \& Kuckuck (2017) anführen, liegen nur wenige Erkenntnisse für den geographischen Kontext vor. Außerdem fehlen für eine Verknüpfung von fachsprachlichem und fachinhaltlichem Lernen vielfach unterrichtspraktische Methoden und Konzepte. Einen möglichen Grund sehen die Autorinnen darin, dass die Verantwortung sprachlicher Förderung oft an die Sprachenfächer abgegeben wurde (vgl. ebd.: 7). Demnach sollte ein Sprachbewusstsein in allen Unterrichtsfächern gestärkt werden.

Budke und Weiss (2014) definieren einen sprachbewussten Fachunterricht als einen Unterricht, der "die fachspezifischen sprachlichen Anforderungen für das Verständnis und zur Beantwortung geographischer Fragestellungen im Unterricht, ausgehend von den Voraussetzungen der Schülerinnen und Schüler berücksichtigt" (ebd.: 127). Das heißt also, die Lehrperson sollte sprachliche Anforderungen, die sich aus der Fachsprache ergeben können, erkennen und entsprechend der sprachlichen Fähigkeiten der Schüler*innen Fördermaßnahmen treffen. Für eine zielgerichtete sprachliche Förderung von heterogenen Lerngruppen wäre es in der Praxis wünschenswert, durch einen Erfahrungsaustausch zwischen den Lehrenden gemeinsame Fördermaßnahmen über die Grenzen einzelner Unterrichtsfächer zu entwickeln.

Für eine solche Entwicklung von systematischen Fördermaßnahmen müssen Grundlagen der sprachlichen Anforderungen berücksichtig werden. Bildungsund Fachsprache stellen spezifische sprachliche Anforderungen an die Lernenden, die sich entlang der drei Ebenen Wort, Satz und Text strukturieren lassen (vgl. Morawski \& Budke 2017: 31). Die Wortebene umfasst geographische und wirtschaftliche Fachbegriffe und deren Verwendung. Die Satzebene bezieht sich auf das Verständnis und die Produktion kommunikativer Sprachhandlungen, die meist eine komplexe Satzstruktur, wie Relativ- oder Konditionalsätze, aufweisen. Die dritten Ebene adressiert die geographische und wirtschaftliche Diskursfähigkeit. Auf dieser Ebene sollen Lernende ein Verständnis für fachtypi- 
sche Textmuster und deren Produktion erwerben, mit dem Ziel das Verständnis für geographische und wirtschaftliche Medien und Themengebiete zu fördern (vgl. Budke \& Kuckuck 2018: 24f.; vgl. Morawski \& Budke 2017: 31).

Mit jeder dieser Ebenen sind bestimmte Sprachhandlungen verbunden, deren Komplexität je nach gefordertem Anforderungsbereich steigt (vgl. Budke \& Kuckuck 2018: 25; Morawski \& Budke 2017: 31). Konkret auf die gängigen Operatoren des Unterrichtsfachs GW übertragen, bedeutet das, dass der Anforderungsbereich I (Reproduktion) die geringsten sprachlichen Anforderungen stellt, während der Anforderungsbereich III (Reflexion und Problemlösung) hohe sprachliche Kompetenzen, wie ein tiefgreifendes, konzeptionelles Verständnis von Begriffen erfordert (vgl. Morawski \& Budke 2017: 31; Bergmeister 2017: 23). Dieser Zusammenhang zwischen Aufgabenschwierigkeit und sprachlichen Anforderungen wirkt sich nachteilig auf Kinder und Jugendliche aus, die nicht über die erforderlichen sprachlichen Kompetenzen verfügen. Für sie ist es deutlich schwieriger Kompetenzen in den höheren Anforderungsbereichen zu erreichen. Das bedeutet keinesfalls den davon betroffenen Schüler*innen Aufgabenstellungen, die einen höheren Anforderungsbereichen adressieren, zu verwehren. Deshalb sind gezielte sprachliche Unterstützungen und Förderungen im Unterricht unabdinglich. Ein sprachförderlicher GW-Unterricht kann und sollte dazu unbedingt einen Beitrag leisten. Das Ziel unserer exemplarischen Schulbuchanalyse besteht darin, potenzielle sprachliche Hindernisse für Schüler*innen aus Autor*innentexten und Aufgaben der GW-Schulbücher herauszufiltern, um das Bewusstsein und die Sensibilität bei Lehrenden zu schärfen. Da es sich um eine exemplarische Studie handelt, verfolgen wir dabei nicht den Anspruch der Repräsentativität.

\section{Forschungsdesign der exemplarischen Schulbuchanalyse}

Im Folgenden soll das Forschungsdesign und damit die forschungsmethodische Herangehensweise der exemplarischen Schulbuchanalyse skizziert werden:

\subsection{Forschungsziel und -frage}

Für die Untersuchung haben wir die Schulbücher GEO_logisch 1, GEOprofi 1 und Durchblick 1 kompetent ausgewählt (Breitfuss-Horner et al. 2020; Mayrhofer et al. 2019; Hofmann-Schneller et al. 2014) ${ }^{3}$.

\footnotetext{
Da wir davon ausgehen, dass Leser*innen aus der Schulpraxis mit den Namen der Schulbuchautorenschaft vielfach nicht das
}

Das Schulbuch GEO_logisch 1 ist erst seit diesem Jahr Teil der Schulbuchaktion. Es handelt sich also um eine Neuerscheinung, die neben der Erklärung von wichtigen (Fach-)Begriffen auch mit der Förderung der Lesekompetenz von Sachtexten wirbt (vgl. Westermann Gruppe 2020a). Die Schulbuchreihe Durchblick der Westermann Gruppe, bietet als einzige Schulbuchreihe ein durchgängiges Konzept von der ersten Klasse der Sekundarstufe I bis zum Abschluss der 8. Klasse der Sekundarstufe II (vgl. Westermann Gruppe 2020b). Das Lehrwerk GEOprofi wird auf der Website des Verlags VERITAS als beliebtestes Lehrwerk der AHS-Unterstufe geführt (vgl. VERITAS o. J.) Alle Werke wurden für die 5. Schulstufe (1. Klasse) der Mittelschule (MS) und AHS Unterstufe approbiert.

Wir haben uns für die 1. Klasse der AHS/ MS entschieden, da wir davon ausgehen, dass die Schüler*innen am Beginn der Sekundarstufe (noch) über wenige Kenntnisse der Fachsprache verfügen und dementsprechend viele neue Fachbegriffe eingeführt werden (müssen). Deshalb adressieren wir auch den ersten Lehrplanbereich „Ein Blick auf die Erde: Erwerben grundlegender Informationen über die Erde mit Globus, Karten, Atlas und Bildern" (BMUKK 2000: 1046), der den Einstieg in das Unterrichtfach GW darstellt. Außerdem lässt sich vermuten, dass am Beginn der Sekundarstufe I die Heterogenität der Lerngruppen im Vergleich zu den anderen Klassenstufen ausgeprägter ist. Entsprechende Hinweise finden sich im nationalen Bildungsbericht Österreich von 2018, der die Sekundarstufe I mit zunehmender Heterogenität charakterisiert (vgl. Braunsteiner et al. 2019: $19 \mathrm{ff}$.).

Aus diesen Überlegungen haben wir folgende Forschungsfrage abgeleitet: Welche potenziellen sprachlichen Hindernisse lassen sich exemplarisch anhand des Schulbuchkapitels, das den Lehrplanbereich "Ein Blick auf die Erde" adressiert, identifizieren? Folgende Kapitel der einzelnen Schulbücher wurden diesem Lehrplanbereich zugeordnet:

- GEOprofi 1: „Unser Planet Erde“ (GEOprofi 1 2019: 6-14) und „Das $1 \times 1$ der Kartenkunde“ (ebd.: 16-26)

- GEO_logisch 1: „Ein erster Blick auf die Erde“ (GEO_logisch 1 2020: 10-13), „Mit Karten arbeiten“ (ebd.: 14-17) und „Geo digital“ (ebd.: 18)

- Durchblick 1 kompetent: „Wir orientieren uns auf der Erde“ (Durchblick 1 kompetent 2014: 6-21)

Schulbuch assoziieren werden, führen wir ab hier nur mehr die Namen der Schulbücher an. Zudem sind viele Personen in den Produktionsprozess eines Schulbuchs involviert, sodass unseres Erachtens nach die wiederholte Nennung der Schulbuchautorenschaft den Fokus zu sehr auf den Autor*innen legt. 


\subsection{Forschungsmethode Herangehensweise}

Für die Beantwortung der Forschungsfrage fand die strukturierende Inhaltsanalyse gemeinsam mit einer Frequenz- bzw. Häufigkeitsanalyse Anwendung. Die Durchführung der einzelnen Analyseschritte erfolgte entsprechend der Vorgangsweise nach Mayring (vgl. 2015: 98), die wir kurz erläutern wollen.

Schritt 1: Als Analyseeinheiten bestimmten wir die Autor*innentexte und Aufgaben der entsprechenden Schulbuchkapitel.

Schritt 2: Mit Hilfe des Leitfadens „Sprache in Schulbüchern" des BMUKK (2012) haben wir folgende Strukturierungsdimensionen (d. h. Hauptkategorien) für die Materialanalyse festgelegt: Wortebene, Satzebene, Textebene und visuelle Ebene.

Wortebene: Die Wortebene beschäftigt sich mit dem Erkennen von Buchstaben und Wörtern und dem Erfassen ihrer Bedeutung (vgl. Chudaske 2012: 80). Dieses semantische Wortverständnis ist grundlegend für den Umgang mit Texten und Aufgabenstellungen in Schulbüchern. Außerdem kann die Wortbedeutung je nach Verwendungskontext veränderbar sein. Es bedarf also eines differenzierten Wortverständnisses seitens der Schüler*innen, um Informationen aus Texten filtern und Aufgabenstellungen erfolgreich bearbeiten zu können. Um diese Herausforderungen zu reduzieren, sollte die Wortverwendung in Schulbüchern präzise und je nach Gebrauchskontext angemessen $\operatorname{sein}^{4}$ (vgl. BMUKK 2012: 12, 16 f.).

Satzebene: Die Satzebene umfasst die syntaktische Struktur von Sätzen und der damit verbundenen semantischen Bedeutung (vgl. Chudaske 2012: 80). Dabei wird zwischen einfachen und komplexen Sätzen unterschieden. Komplexe Sätze lassen sich in zwei Kategorien einteilen: „Parataxe (Nebenordnung gleichrangiger Teilsätze) und [...] Hypotaxe (Unterordnung ungleichrangiger Teilsätze)“ (BMUKK 2012: 13). Je komplexer der Satzbau, umso höher sind die sprachlichen Anforderungen für Lernende (vgl. ebd.: 13).

\footnotetext{
Allerdings steht die kontextadäquate Wortverwendung auch im Zusammenhang mit dem fachlichen Vorwissen und Konzeptverstehen der Schüler*innen. Lehrpersonen sind daher gefordert die Voraussetzungsketten ihrer Schüler*innen, die im Zusammenhang mit dem Verständnis bestimmter Begriffe stehen, zu erkennen. Nur dadurch kann eine Ausgangsbasis für neue Verstehens- und fachliche Vernetzungsprozesse geschaffen werden. D. h. Begriffe auf der Wortebene, die sich auf komplexe fachliche Konzepte beziehen, wie zum Beispiel der Begriff „Raum“, bedürfen hierbei besonderer Aufmerksamkeit (vgl. Bergmeister 2017: 18 ff.).
}

Textebene: Auf der Textebene werden Sätze zu Bedeutungseinheiten verknüpft, wodurch „eine mentale Repräsentation der Textbedeutung " aufgebaut wird (Chudaske 2012: 80). Der Zusammenhang zwischen den einzelnen Sätzen eines Textes kann durch sprachliche Hilfen, wie Kohäsionsmittel, unterstützt werden. Dadurch werden die verknüpften Informationen für Lernende nachvollziehbarer. Allerdings braucht es auch außersprachliches Wissen (z. B. Weltwissen, Handlungswissen), um einen Sinnzusammenhang zwischen den Informationen erkennen zu können (vgl. BMUKK 2012: 14). „Dieses Wissen kann kulturell unterschiedlich geprägt sein und bei Kindern mit Deutsch als Zweitsprache zu Verständnisproblemen führen“ (ebd.: 14).

Visuelle Ebene: Die visuelle Ebene dient der bildlichen und graphischen Veranschaulichung von Informationen. „Ein Zweck von Visualisierungen besteht daher darin, das inhaltliche Verständnis von Texten zu erleichtern" (BMUKK 2012: 15), beispielsweise durch das Hervorheben von wichtigen Informationen. Dadurch können Schüler*innen zentrale Inhalte leichter erkennen und ihr Textverständnis wird unterstützt (vgl. ebd.: 15).

Schritt 3: Wie Tabelle 1 zeigt, besteht das Kategoriensystem aus diesen vier Hauptkategorien sowie ihren jeweiligen verschiedenen Unterkategorien. Die Kapitel wurden entsprechen des Kategoriensystems untersucht.

Schritt 4. und 5: Es erfolgten mehrmalige Materialdurchläufe und das Kodierschema wurde wiederholt überarbeitet. Dabei verwendeten wir zwei Kodierschemata: eines für die Autor*innentexte und eines für die Aufgaben. Diese zwei Kodierschemata werden in Tabelle 1 fusioniert dargestellt. Die Fundstellen der potenziellen sprachlichen Hürden wurden in den Schulbüchern je nach Kategorie farblich markiert. Der nachstehende Kodierleitfaden beinhaltet die vier Hauptkategorien, den theoretischen Hintergrund, das Farbschema, die Definition der Kategorie, sowie Ankerbeispiele aus dem Material. Im Bereich der visuellen Ebene wurde im Bereich der Ankerbeispiele teilweise auf einzelne Seiten verwiesen, da sich die Beispiele nur schwer in einer Tabelle darstellen lassen. Für einzelne Unterkategorien konnten keine Ankerbeispiele im Material gefunden werden. Dies wurde ebenfalls vermerkt.

Schritt 6: Nach der Kodierung des Materials haben wir die Fundstellen zum Großteil wortwörtlich je nach Kategorie in eine Tabelle übertragen. Diese Tabelle stellte die Grundlage für der anschließenden Frequenz- bzw. Häufigkeitsanalyse dar. 
Tab. 1: Kodierschema (eigene Darstellung; Herv. d. V.)

\begin{tabular}{|c|c|c|c|c|}
\hline $\begin{array}{l}\text { Kate- } \\
\text { gorie }\end{array}$ & $\begin{array}{l}\text { Theoretischer Hintergrund zur } \\
\text { Identifikation sprachlicher Hürden }\end{array}$ & $\begin{array}{l}\text { Farb- } \\
\text { code }\end{array}$ & $\begin{array}{l}\text { Definition der Kategorie } \\
\text { (vgl. BMUKK 2012: } 7 \text { ff.) }\end{array}$ & Ankerbeispiele aus dem Material \\
\hline 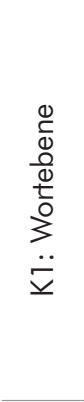 & $\begin{array}{l}\text { Besonders für Schüler*innen mit } \\
\text { Deutsch als Zweitsprache sind } \\
\text { eine Vielzahl an neu eingeführten } \\
\text { Wörtern (Fremdwörter), (komplexe) } \\
\text { Komposita und Fachbegriffe oft } \\
\text { problematisch. Daher sollte deren } \\
\text { Bedeutung präzise und verständlich } \\
\text { erklärt werden und die Verwendung } \\
\text { in mehreren Gebrauchszusam- } \\
\text { menhängen erfolgen (vgl. BMUKK } \\
\text { 2012: } 12 \text { ff.). }\end{array}$ & ROT & $\begin{array}{l}\text { Die Kategorie der } \\
\text { Wortebene beinhaltet } \\
\text { (komplexe) Komposita }^{5} \text {, } \\
\text { Fremdwörter und Fach- } \\
\text { wörter, die nicht präzise } \\
\text { und verständlich erklärt } \\
\text { werden, sowie ambige } \\
\text { Wörter/Wortverwendun- } \\
\text { gen und Nominalisie- } \\
\text { rungen mittels Derivation } \\
\text { und Konversion. }\end{array}$ & $\begin{array}{l}\text { (komplexe) Komposita: "Hauptsiedlungsraum" } \\
\text { (S. } 12 \text { in GEOprofi 1) } \\
\text { Fremdwörter: "Global Positioning System" } \\
\text { (S. } 10 \text { in Durchblick 1 kompetent) } \\
\text { Fachwörter: "Baumwollplantagen" } \\
\text { (S. } 24 \text { in GEOprofi 1) } \\
\text { Ambige Wortverwendung: "während" } \\
\text { (S. } 6 \text { in GEOprofi 1) } \\
\text { Nominalisierungen: "das Schrumpfen" } \\
\text { (S. } 24 \text { in GEOprofi 1) }\end{array}$ \\
\hline 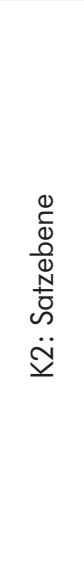 & $\begin{array}{l}\text { Das Aufgaben- und Textver- } \\
\text { ständnis der Schüler*innen kann } \\
\text { durch komplexe Sätze, damit sind } \\
\text { hypotaktische und parataktische } \\
\text { Strukturen gemeint, sowie durch } \\
\text { Passivkonstruktionen und unvoll- } \\
\text { ständige Sätze erschwert werden. } \\
\text { Vor allem komplexe Sätze sollten } \\
\text { nicht vollständig vermieden werden, } \\
\text { sie sollten jedoch in ihrer Komple- } \\
\text { xität für die Schulstufe angemessen } \\
\text { sein, um die wesentlichen Informa- } \\
\text { tionen herausfiltern zu können (vgl. } \\
\text { BMUKK 2012: } 13 \text { ff.). }\end{array}$ & GRÜN & $\begin{array}{l}\text { Die Kategorie der Sat- } \\
\text { zebene beinhaltet kom- } \\
\text { plexe hypotaktische }{ }^{6} \text { und } \\
\text { parataktische }{ }^{7} \text { Satzstruk- } \\
\text { turen, Passivkonstruktio- } \\
\text { nen und unvollständige } \\
\text { Sätze sowie das Fehlen } \\
\text { von Operatoren in Auf- } \\
\text { gabenstellungen. }\end{array}$ & $\begin{array}{l}\text { Komplexe hypotaktische/ parataktische Satz- } \\
\text { strukturen: } \\
\text { "An den unterschiedlichen Farben der Land- } \\
\text { massen erkennt man, ob sich dort große Wald- } \\
\text { flächen oder trockene Gebiete wie zum Beispiel } \\
\text { Wüsten befinden oder ob es Schnee gibt" } \\
\text { (S. } 10 \text { in GEO logisch 1) } \\
\text { Passivkonstruktionen: "Menschen leben in der } \\
\text { Antarktis keine, es werden aber Forschungsstati- } \\
\text { onen betrieben" (S. } 10 \text { in GEOprofi 1; Herv. d. V.) } \\
\text { Unvollständige Sätze: "Zum Kontinent Austra- } \\
\text { lien zählen auch eine Reihe von Inseln im Pa- } \\
\text { zifischen Ozean, [sie/diese werden] Ozeanien } \\
\text { genannt" (S. } 10 \text { in GEOprofi 1; Herv. d. V.) } \\
\text { Fehlen von Operatoren: Verwendung von Ope- } \\
\text { ratoren in allen Aufgabenstellungen }\end{array}$ \\
\hline 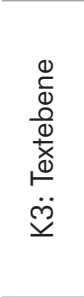 & $\begin{array}{l}\text { Für Textinterpretationen ist außer- } \\
\text { sprachliches Wissen erforderlich. } \\
\text { Daher sollten Kohäsionsmittel } \\
\text { verwendet werden, um Sinnzusam- } \\
\text { menhänge logisch nachvollziehbar } \\
\text { zu gestalten (vgl. BMUKK 2012: 14). }\end{array}$ & GELB & $\begin{array}{l}\text { Textpassagen, deren } \\
\text { Sätze ohne Kohäsions- } \\
\text { mittel aneinandergereiht } \\
\text { wurden, sowie Aufga- } \\
\text { benstellungen, in denen } \\
\text { nötige Informationen zur } \\
\text { Bearbeitung fehlen. }\end{array}$ & $\begin{array}{l}\text { Fehlen von Kohäsionsmittel: } \\
\text { "Österreich würde mehr als hundertmal in die } \\
\text { Sahara passen. Auf dem Kontinent Afrika finden } \\
\text { wir noch einen zweiten Rekord:[...]" (S. } 12 \text { in } \\
\text { GEOprofi 1) } \\
\text { Nötige Informationen fehlen: } \\
\text { Es wurden keine Beispiele im Bereich der Auf- } \\
\text { gaben gefunden. }\end{array}$ \\
\hline 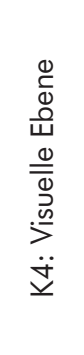 & $\begin{array}{l}\text { Optische Hervorhebungen von } \\
\text { wichtigen Begriffen in Texten können } \\
\text { Vorwissen aktivieren und damit } \\
\text { inhaltliches Verständnis erleichtern. } \\
\text { Absätze und Aufzählungen ver- } \\
\text { deutlichen Zusammenhänge von } \\
\text { Informationen. Ungegliederte Texte } \\
\text { können motivations- und verständ- } \\
\text { nishinderlich sein (vgl. BMUKK } \\
\text { 2012: } 34 \text { f.). }\end{array}$ & BLAU & $\begin{array}{l}\text { Textpassagen, die nicht } \\
\text { gegliedert sind, das } \\
\text { heißt Aufzählungen oder } \\
\text { Absätze fehlen. Wichtige } \\
\text { Begriffe/Inhalte sind } \\
\text { nicht hervorgehoben. }\end{array}$ & $\begin{array}{l}\text { Fehlen von Aufzählungen und Absätzen } \\
\text { (S. } 6 \text { in GEOprofi 1) } \\
\text { Wichtige Begriffe/Inhalte werden nicht hervor- } \\
\text { gehoben (S. } 12 \text { in GEOprofi 1) }\end{array}$ \\
\hline
\end{tabular}

\subsection{Quantitative Analyse}

Die Frequenz- bzw. Häufigkeitsanalyse (vgl. Mayring 2015: 15) der potenziellen sprachlichen Hürden umfasste alle vier Hauptkategorien, sowie eine Zählung und Häufigkeitsdarstellung der drei Anforderungsbereiche der Aufgabenstellungen. Eine quantitative

\footnotetext{
Ausgenommen sind Ortsbezeichnungen, wie „Nordeuropa“, oder Zwischenhimmelsrichtungen, wie „Nordnordwest“.

6 „Bei einer Hypotaxe (Satzgefüge) ist ein Teilsatz dem anderen untergeordnet (subordiniert)" (Pittner \& Berman 2015: 96). - Beispiel: „So verwundert es kaum, dass die riesige Insel nur ca. 60000 Einwohner zählt" (GEOprofi 1 2019: 12).
}

Analyse der drei Anforderungsbereiche wurde aufgrund des bereits erwähnten Zusammenhangs zwischen höheren Anforderungsbereichen und höherem sprachlichen Niveau bei der Bearbeitung der Aufgaben durchgeführt (vgl. Budke \& Kuckuck 2018: 25; vgl. Morawski \& Budke 2017: 31). Das Verfahren ermöglicht zudem einen Vergleich der Häufigkeit des Auftretens potenzieller sprachlicher Hürden auf den

„Bei einer Parataxe (Satzreihung) werden strukturell gleichrangige Sätze verbunden. Dabei kann es sich um Hauptsätze handeln, aber auch um Nebensätze" (Pittner \& Berman 2015: 96). Beispiel: „Bestimme das jeweilige Planquadrat und schreibe es neben die Orte" (GEO_logisch 1 2020: 16). 
verschiedenen Ebenen (Wort-, Satz-, Text- und visuelle Ebene). Außerdem kann dargestellt werden mit welcher Häufigkeit die drei Anforderungsbereiche in den Aufgabenstellungen vertreten sind.

\section{$4 \quad$ Ergebnisdarstellung}

\subsection{Ergebnisse - Autor*innentexte}

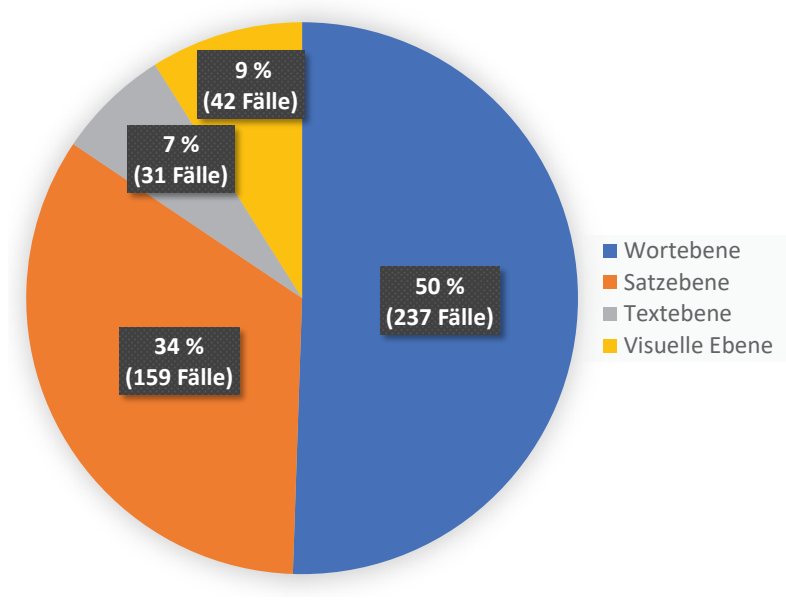

Abb. 1: Potenzielle sprachliche Hindernisse in den Autor*innentexten (Quelle: eigene Darstellung)

Insgesamt konnten wir in den Autor*innentexten 469 potenzielle sprachliche Hindernisse identifizieren (siehe Abb. 1). Der Großteil (50\%) betrifft die Wortebene (237 Fälle) und entfällt auf die Unterkategorie (komplexe) Komposita (201 Fälle), wie beispielsweise "Oberflächentemperatur" (GEOprofi 1 2019: 8), "Hauptsiedlungsraum“ (ebd.: 12), „Fischreichtum “(ebd.: 24), „Inneneinband“(GEO_logisch 1 2020: 14), sowie „Berghöhen“(Durchblick 1 2014: 9). Mit 8 Fundstellen zeigt sich, dass Fremd- und Fachbegriffe, wie beispielsweise „Baumwollplantagen“ und „Rinnsälen“ GEOprofi 1 (2019: 24), kaum sprachliche Hindernisse darstellen, weil sie meist erklärt und dann auch mehrfach verwendet werden. Auch im Bereich der ambigen Wörter und Wortbedeutungen lassen sich nur einige wenige Beispiele (5 Fälle) finden. Ein konkretes Beispiel dafür findet sich im Schulbuch GEOprofi 1 (2019: 6): „Am Merkur kann es etwa bis $z$ zu $430^{\circ} \mathrm{C}$ heiß werden, während die Temperatur am Neptun auf $-200^{\circ} \mathrm{C}$ absinken kann!" - Herausfordernd ist hier vor allem die Wortbedeutung der Konjunktion "während", da sie sowohl temporal als auch adversativ verwendet werden kann.

Schlussendlich konnten in der Kategorie Wortebene auch Nominalisierungen (23 Fälle) als sprachliche Hürden identifiziert werden. Beispiele hierfür wären: "Versalzen“(GEOprofi 1 2019: 24), „das Schrumpfen“" (ebd.: 24), „beim Bergwandern“ (Durchblick 1 kompetent 2014: 13) und „Verkleinerung“(ebd.: 14).

In der Kategorie Satzebene identifizierten wir 159 Fundstellen, entsprechend 34\% der gesamten aufgefundenen potenziellen sprachlichen Hürden $(\mathrm{N}=469)$. Dabei achteten wir insbesondere auf komplexe hypotaktische und parataktische Satzstrukturen, wie beispielsweise Relativ- oder Konditionalsätze, die den Großteil, konkret 149 Fundstellen, ausmachen. "Wir müssen auf unsere Erde gut achtgeben, denn auf andere Planeten unseres Sonnensystems können wir aufgrund der dort vorherrschenden lebensfeindlichen Bedingungen nicht ausweichen " (GEOprofi 1 2019: 6). In der Unterkategorie Passivkonstruktionen fanden wir insgesamt 8 Fundstellen, wie beispielsweise „Menschen leben in der Antarktis keine, es werden aber Forschungsstationen betrieben" (GEOprofi 1 2019: 10). Die Unterkategorie der unvollständigen Sätze umfasst mit zwei Fällen die wenigsten Fundstellen. Ein Beispiel hierfür wäre: „Deshalb heißt sie auch Seehöhe, manchmal [nennt man sie] auch Höhe über Normalnull (NN)"(Durchblick 1 2014: 13; Herv. d. V.).

Auf der Textebene ließen sich 7\%, also 31 Fälle identifizieren. Im Allgemeinen sind die Schulbuchtexte sehr kohäsiv geschrieben. Ein Beispiel für einen nicht einfach nachvollziehbaren Gedankengang, da hier außersprachliches Wissen für das Verständnis unabdinglich ist: „Bei Vulkanausbrüchen dringt die Gesteinsschmelze bis an die Erdoberfläche vor. Der Erdkern schließlich besteht aus Metallen wie Eisen und Nickl" (GEOprofi 1 2019: 8).

In der vierten Kategorie der visuellen Ebene traten 9\% (entspricht 42 Fällen) der Sprachschwierigkeiten auf. Das analysierte Kapitel im Schulbuch GEOprofi 1 (2019) ist durch inkonsistente grafische Gestaltungselemente charakterisierbar. Auf einzelnen Seiten sind wichtige (Fach-)Begriffe in fett hervorgehoben, während andere Seiten keine Hervorhebungen aufweisen und bei einer hohen Textdichte vielfach Absätze fehlen (vgl. ebd.: 6). Damit fehlen weitgehend Strukturen, die für die Überschaubarkeit der Textmenge wichtig wären. Diese Problematik konnten wir für Durchblick 1 kompetent (2014) und GEO_logisch 1 (2020) nicht feststellen.

\subsection{Ergebnisse - Aufgaben}

Die Gesamtzahl von 293 potenziellen sprachlichen Hindernissen bei den Aufgaben weist eine ähnliche Verteilung zu jener der Autor*innentexte auf (siehe Abb. 2). Ausgenommen davon sind die 77 Fälle der Kategorie der visuellen Ebene. Im analysierten Kapitel von GEOprofi 1 (2019) zeigt sich die Problematik der Visualisierung auch bei den Aufgaben. Abgesehen von den Überschriften, gibt es keine visuellen Hilfsmittel. 


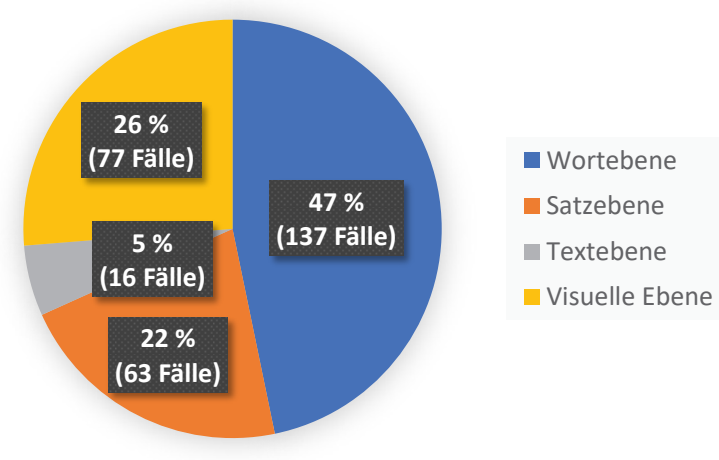

Abb. 2: Potenzielle sprachliche Hindernisse in den Aufgaben (Quelle: eigene Darstellung)

Zudem fehlen bei der Aufgabe auf Seite 7 die Nummerierungen der Teilaufgaben. Nur im Schulbuch GEO_logisch 1 (2020) werden bei den Aufgaben teilweise wichtige (Fach-)Begriffe in fett hervorgehoben. Jedoch bezieht sich das nur auf jene Begriffe, die auch erklärt werden. Eine durchgängige Markierung von wichtigen Fachbegriffen findet also nicht statt.

Der Großteil der potenziellen sprachlichen Hindernisse betreffen wiederrum die Wortebene $(47 \%=$ 137 Fundstellen). Auch hierbei sind diese hauptsächlich der Unterkategorie der (komplexen) Komposita (122 Fälle) zuzuordnen, beispielsweise „Sonnenscheindauer" (GEOprofi 1 2019: 14), „Nutztierhaltung“" (ebd.: 21), "Jahresniederschlag" (Durchblick 1 kompetent 2014: 19), „Kartennutzung“ (GEO_logisch 1 2020: 18). In den anderen Unterkategorien der Wortebene lassen sich weniger Beispiele finden. Beispiele für nicht erklärte Fach- und Fremdwörter (7 Fälle) sind „Navigieren“ (GEO_logisch 1 2020: 18) oder „Nutztierhaltung“ (GEOprofi 1 2019: 21). Der Begriff „Maus “ fällt in die Unterkategorie ambiges Wort (4 Fälle) (Durchblick 1 kompetent 2019: 20; GEO_ logisch 1 2020: 18). In beiden Schulbüchern ist damit nicht das Nagetier, sondern die Computermaus gemeint, was jedoch aus dem Kontext klar hervorgeht. Nominalisierungen werden in den Aufgaben (4 Fälle) nur in Ausnahmefällen verwendet, wie etwa "Vergrößerung"(GEOprofi 1 2019: 26).

Die Satzebene stellt bei den Aufgaben 22\% (63 Fälle) der potenziellen Probleme dar und betrifft hauptsächlich die Unterkategorie der komplexen Sätze (62 Fundstellen), wie beispielsweise: „Überlege, was bei deinem Weihnachtsfest ablaufen würde, wenn $d u$ auf der Südhalbkugel wohnen würdest" (GEOprofi 1 2019: 9). Zudem lässt sich nur eine Fundstelle für die Unterkategorie Passivkonstruktionen identifizieren: "Finde heraus, welche Kontinente hier beschrieben werden "(Durchblick 1 kompetent 2014: 8). Für alle anderen Unterkategorien gibt es keine Fundstellen.

Auf der Textebene traten mit 16 Fällen, entsprechend 5\% der Gesamtergebnisse, die wenigsten sprachlichen Hindernisse auf. Für die Bearbeitung der Aufgaben sind in allen Fällen die nötigen Informationen vorhanden.

\subsection{Analyse der Anforderungsbereiche}

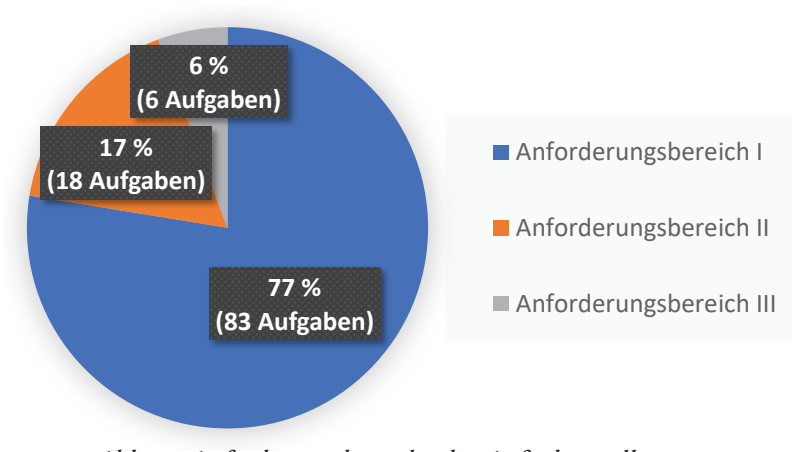

Abb. 3: Anforderungsbereiche der Aufgabenstellungen (Quelle: eigene Darstellung) ${ }^{8}$

Die Frequenz- bzw. Häufigkeitsanalyse der Anforderungsbereiche der Aufgabenstellungen ergab, wie Abb. 3 zeigt, folgendes Bild: $77 \%$, also 83 Aufgaben, der insgesamt 107 Aufgabenstellungen in den drei Schulbüchern erfordern den Anforderungsbereich I der Reproduktion. $17 \%$, also 18 Aufgabenstellungen, adressieren den Anforderungsbereich II Reorganisation und Transfer, während 6\% (6 Aufgaben), den Anforderungsbereich III Reflexion und Problemlösung betreffen.

Ein differenzierteres Bild ergibt die Auswertung der Verteilung der Anforderungsbereiche untergliedert nach den einzelnen Schulbüchern (siehe Abb. 4).

Es zeigt sich, dass im Schulbuch GEOprofi 1 (2019) $92 \%$ der insgesamt 24 Aufgaben den Anforderungsbereich I erfordern. $8 \%$ (also 2 Aufgaben) sind dem Anforderungsbereich II zuordenbar und keine einzige Aufgabe gehört dem Anforderungsbereich III an.

Im Schulbuch Durchblick 1 kompetent (2014) konnten wir für die analysierten Kapitel 37 Aufgabenstellungen zählen. Die Anforderungsbereiche werden in einer Verteilung von $57 \%$ (Anforderungsbereich I), $32 \%$ (Anforderungsbereich II) und 11\% (Anforderungsbereich III) adressiert.

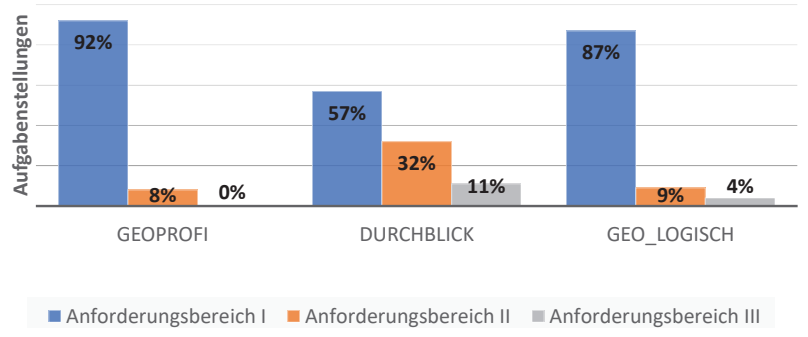

Abb. 4: Anforderungsbereiche der Aufgabenstellungen je Schulbuch (Quelle: eigene Darstellung)

8 Für die Darstellung der Anforderungsbereiche haben wir uns am Schema der Operatoreneinteilung nach Sitte 2011 orientiert. 
Im Schulbuch GEO_logisch 1 (2020) betrifft ebenfalls ein Großteil, nämlich 40 Aufgaben, der insgesamt 46 Aufgaben den Anforderungsbereich I (87\%). Auf den Anforderungsbereiche II fallen 4 Aufgaben (9\%) und 2 Aufgaben (4\%) erfordern den Anforderungsbereich III.

\section{Ergebnisdiskussion und Zusammenfassung}

Zusammengefasst ergibt sich für die exemplarische Schulbuchuntersuchung, dass wir in den nach Autor*innentexten und Aufgaben getrennten, mehrfachen Analysedurchgängen insgesamt 762 potenzielle sprachliche Hindernisse identifizieren konnten. Das Adjektiv potenziell verweist zwar nur auf eine Möglichkeit, jedoch ist die Anzahl angesichts dessen, dass wir nur einzelne Kapitel untersucht haben, dennoch beträchtlich. Der Großteil betrifft (komplexe) Komposita auf Wortebene. Komposita sind eine Eigenheit der deutschen Sprache, deren Komplexität sich möglicherweise Personen mit Deutsch als Erstsprache nicht bewusst sind.

Kritisch möchten wir anmerken, dass die Satzstruktur in den ausgewählten GW-Schulbüchern oft sehr komplex ist und viele Schachtelsätze verwendet werden. Bei der Analyse war besonders auffällig, dass im Schulbuch GEOprofi 1 die Komplexität der Satzstrukturen vom Beginn (S. 6) bis hin zum Ende abnimmt. Dies spricht explizit gegen die Empfehlung des BMUKK (2012: 13), komplexe Satzstrukturen zwar zu verwenden, ihre Komplexität jedoch kontinuierlich zu steigern.

Auf Textebene ergeben sich wenige potenzielle sprachliche Probleme, da die Schulbücher im Allgemeinen viele Kohäsionsmittel verwenden, um einen Zusammenhang zwischen einzelnen Sätzen und den darin vorhandenen Informationen herzustellen.

Defizite auf der visuellen Ebene zeigten sich vor allem bei den Aufgaben der Schulbücher. Hier wurden kaum Informationen hervorgehoben. Bei den Autor*innentexten ist die Darstellungsweise des Schulbuchs GEOprofi 1 kritisch anzusehen, da es sehr wenige oder auf einzelnen Seiten auch gar keine visuellen Hervorhebungen beinhaltet und somit visuelle Orientierungsmittel vielfach fehlen.

Die Verteilung der Anforderungsbereiche ist in den analysierten Schulbuchkapiteln unterschiedlich ausgeprägt. Einzig das Schulbuch Durchblick 1 kompetent umfasst im Kapitel „Wir orientieren uns auf der Erde" mehrere Aufgabenstellungen, die über die Ebene der Reproduktion hinausgehen. GEO_logisch 1 und GEOprofi 1 fokussieren dabei verhältnismäßig stark auf den Anforderungsbereich I. Im Schulbuch GEOprofi gibt es zudem keine einzige Aufgabenstel- lung für den Anforderungsbereich III und nur zwei Aufgaben für den Anforderungsbereich II. Die geringe Berücksichtigung höherer Anforderungsbereiche ist fachdidaktisch bedenklich, denn laut Leistungsbeurteilungsverordnung (LBVO) erfordern die Noten "Gut" und „Sehr gut" seitens der Lernenden eigenständiges Arbeiten und selbstständiges Anwenden des eigenen Wissens und Könnens. Bieten Schulbücher jedoch Aufgaben an, die sich hauptsächlich im Anforderungsbereich I befinden, also auf die Reproduktion von Wissen abzielen, besteht diese selbstständige Anwendung auf neuartige Aufgaben nicht. Folglich wären solche Leistungen im Bereich der Note „Befriedigend“ oder darunter einzuordnen (vgl. BMUK 1974: 10). Zudem sollte die Vielzahl an Aufgabenstellungen im Anforderungsbereich I auch kritisch gesehen werden, da die reine Reproduktion von Wissen dazu führen kann, dass Begrifflichkeiten unreflektiert wiedergeben und ihre Verwendungs- und Bedeutungszusammenhänge für die Lebenswelten der Lernenden unklar bleiben. Vielhaber (1999) spricht diesbezüglich von „totem Wissen“ (vgl. Vielhaber 1999: 13).

Allerdings stellen Aufgabenstellungen der Reflexion und Problemlösung auch höhere sprachliche Anforderungen an die Lernenden, deshalb wären an diesen Stellen geeignete Methoden und Hilfestellungen wichtig. In keinem der drei Schulbücher gibt es dafür unterstützende sprachliche Maßnahmen wie beispielsweise das Bereitstellen von Satzanfängen für Argumentationen oder auch Musterlösungen, an denen sich Schüler*innen orientieren könnten. Zusammenfassend ergibt sich, dass sich auf allen Ebenen sprachlicher Anforderung und auch auf der visuellen Ebene potenzielle sprachliche Schwierigkeiten für Schüler*innen heterogener Lerngruppen feststellen lassen. Dabei gilt es zu berücksichtigen, dass es sich lediglich um potenzielle sprachliche Schwierigkeiten handelt, die in ihrer Anwendung nicht komplett vermieden werden sollten. Jedoch sollten sie kontextadäquat und unter Berücksichtigung der sprachlichen Voraussetzungen der einzelnen Schüler*innen eingesetzt werden.

\section{$6 \quad$ Fazit}

Die Analyse zeigt deutlich, dass die analysierten Schulbuchkapitel eine Vielzahl an potenziellen sprachlichen Hindernissen aufweisen. Dessen sollten sich Lehrpersonen bewusst sein, wenn sie diese im Unterricht mit heterogenen Lerngruppen verwenden, um entsprechende sprachsensible zusätzliche Maßnahmen zu setzen.

Um die Schüler*innen zu unterstützen und den Unterricht sprachsensibel zu gestalten, können ver- 
schiedene Methoden, wie die Scaffolding-Prinzipien (Sprachlerngerüste), eingesetzt werden. Dazu zählen beispielsweise Wortlisten oder Erklärungen, um die Formulierung von komplexen Sachverhalten zu vereinfachen (vgl. Carnevale \& Wojnesitz 2014: 14). Gerade beim Lösen von Aufgabenstellungen kommt dieser Methode besondere Bedeutung zu. Ausgehend von den Erkenntnissen der vorliegenden Schulbuchanalyse haben wir entsprechende unterrichtspraktische Empfehlungen gestaltet, die aufzeigen, wie sprachsensibles Unterrichten im GW-Unterricht umsetzbar wäre (siehe Praxisbeitrag von Anich \& Stieger). Denn wie bereits diskutiert, ist der Erwerb und die Verbesserung (bildungs-)sprachlicher Kompetenzen nicht alleinige Aufgabe der Sprachfächer, sondern ein verpflichtender Bestandteil aller Unterrichtsfächer, so auch des GW-Unterrichts.

Abschließend möchten wir festhalten, dass sich Schulbücher im Bewusstsein ihrer sprachlichen Hürden als Unterrichtsmedium für heterogene Lerngruppen durchaus eignen, aber Lehrpersonen durch gezielten Methodeneinsatz des sprachsensiblen Unterrichtens beim Umgang mit diesem Medium unterstützend tätig sein sollten, um allen Lernenden die Möglichkeit zu geben auch Aufgabenstellungen höherer Anforderungsbereiche zu bewältigen.

\section{Dank}

Wir danken unseren beiden anonymen Gutachter*innen für ihre konstruktiven Kommentare und Verbesserungsvorschläge sowie der Pädagogischen Hochschule Stefan Zweig für die Unterstützung dieser Publikation.

\section{Literatur}

Bergmeister, F. M. (2017): Schwellenkonzepte als Zugänge fachlichen Verstehens - Wege zur differenzierten Erschließung komplexer (ökonomischer) Basiskonzepte im GW-Unterricht. In: GW Unterricht 147. S. 16-25. DOI: https://doi.org/10.1553/gw-unterricht147s16

Breitfuss-Horner, C., C. Sitte, I. Sprenger \& R. Zölfel (2020): GEO_logisch 1: Geographie und Wirtschaft. Westermann, Wien. S. 10-18.

Braunsteiner, M. L., C. Fischer, G. Kernbichler, A. Prengel \& D. Wohlhart (2019): Erfolgreich lernen und unterrichten in Klassen mit hoher Heterogenität. In: Breit, S., F. Eder, K. Krainer, C. Schreiner, A. Seel \& C. Spiel (Hrsg.): Nationaler Bildungsbericht Österreich 2018: Fokussierte Analysen und Zukunftsperspektiven für das Bildungswesen. Leykam, Graz. S. 19-62.

Budke, A. \& M. Kuckuck (2017): Sprache im Geographieunterricht. In: Budke, A. \& M. Kuckuck (Hrsg.): Sprache im Geographieunterricht: Bilinguale und sprachsensible Materialien und Methoden. Waxmann, Münster. S. 7-35.
Budke, A. \& M. Kuckuck (2018): Migration und geographische Bildung. In: Budke, A. \& M. Kuckuck (Hrsg.): Migration und geographische Bildung. Franz Steiner, Stuttgart. S. 9-36.

Budke, A. \& G. Weiss (2014): Sprachsensibler Geographieunterricht. In: Michalak, M. (Hg.): Sprache als Lernmedium in allen Fächern. Schneider, Baltmannsweiler. S. $113-133$.

BMUK - Bundesministerium für Unterricht und Kunst (1974): Verordnung des Bundesministers für Unterricht und Kunst vom 24. Juni 1974 über die Leistungsbeurteilung in Pflichtschulen sowie mittleren und höheren Schulen (Leistungsbeurteilungsverordnung). https://www.ris. bka.gv.at/GeltendeFassung/Bundesnormen/10009375/ Leistungsbeurteilungsverordnung $\% 2 \mathrm{c} \% 20$ Fassung $\% 20$ vom\%2019.02.2021.pdf (29.03.2021)

BMUKK - Bundesministerium für Unterricht, Kunst und Kultur (2000): Lehrplan der AHS-Unterstufe für Geographie und Wirtschaftskunde. Bildungs- und Lehraufgabe. In: Bundesgesetzblatt II Nr. 133 v. 11.05.2000; S. 1044-1048 https://www.ris.bka.gv.at/Dokumente/BgblPdf/2000_133_2/2000_133_2.pdf (29.03.2021)

BMUKK - Bundesministerium für Unterricht, Kunst und Kultur (2012): Sprache in Schulbüchern: Empfehlungen zur Sprachverwendung in Schulbüchern für SchulbuchautorInnen, GutachterInnen und Schulbuchverlage. Digitales Druckzentrum des BMUKK, Wien.

BMBWF - Bundesministerium für Bildung, Wissenschaft und Forschung (2017): Grundsatzerlass Leseerziehung. https://www.bmbwf.gv.at/Themen/schule/schulpraxis/ prinz/leseerziehung.html (29.03.2021)

Carnevale, C. \& A. Wojnesitz (2014): Sprachsensibler Fachunterricht in der Sekundarstufe: Grundlagen - Methoden - Praxisbeispiele. In: ÖSZ Praxisreihe 23. ÖSZ, Graz.

Chudaske, J. (2012): Sprachliche Kompetenz. In: Chudaske, J. (Hg.): Sprache, Migration und schulfachliche Leistung Einfluss sprachlicher Kompetenz auf Lese-, Rechtschreibund Mathematikleistungen. VS, Wiesbaden. S. 37-102.

Hintermann, C., C. Markom, H. Weinhäupl \& S. Üllen (2014): Debating Migration in Textbooks and Classrooms in Austria. In: Journal of Educational Media, Memory, and Society 6. S. 79-106. DOI: https://doi. org/10.3167/jemms.2014.060105

Hofmann-Schneller, M., F. Graf, W. Scheidl \& K. Steiner (2014): Durchblick 1 kompetent: Geographie und Wirtschaftskunde für die 5. Schulstufe. Westermann, Wien. S. 6-21.

Lehrplankommission GW (2021): Geographie und wirtschaftliche Bildung, Lehrplanentwurf Nr. 6 v. 15.03.2021. Wien. https:/www.eduacademy.at/gwb/ $\mathrm{mod} /$ resource/view.php?id=30002 (29.03.2021)

Mayrhofer, G., R. Posch \& I. Reiter (2019): GEOprofi 1: Geographie und Wirtschaftskunde für die 5. Schulstufe (2. Aufl.). Veritas, Linz. S. 6-14; S.16-26.

Mayring, P. (2015): Qualitative Inhaltsanalyse. Grundlagen und Techniken. Beltz, Weinheim. 
Morawski, M. \& A. Budke (2017): Sprachförderkonzepte in bilingualen Geographieschulbüchern. In: GW Unterricht 148. S. 28-42. DOI: https://doi.org/10.1553/gwunterricht 148 s 28

Pittner, K. \& J. Berman (2015): Deutsche Syntax: Ein Arbeitsbuch (6.Aufl.). Narr, Tübingen.

Schroeter-Brauss, S., V. Wecker \& L. Henrici (2018): Sprache im naturwissenschaftlichen Unterricht: Eine Einführung. Waxmann, Münster.

Sitte, C. (2001): Das GW-Schulbuch. In: Sitte, W. \& H. Wohlschlägl (Hrsg.): Beiträge zur Didaktik des „Geographie und Wirtschaftskunde“-Unterrichts. Institut für Geographie und Regionalforschung, Wien. S. 447-472.

Sitte, C. (2011): Maturafragen NEU (!?) - eine schrittweise Annäherung an eine kompetenzorientierte Form in Geographie und Wirtschaftskunde, Anhang 4: Operatorenliste. In: GW-Unterricht 123. S. 39-41. http://www. gw-unterricht.at/images/pdf/gwu_123_024_041_sitte. pdf (17.02.2021)
VERITAS (o. J.): GEOprofi. https://www.veritas.at/reihe/ geoprofi (17.02.2021)

Vielhaber, C. (1999): Vermittlung und Interesse. Zwei Schlüsselkategorien fachdidaktischer Grundlagen im Geographieunterricht. In: Vielhaber, C. (Hg.): Geographiedidaktik kreuz und quer. Vom Vermittlungsinteresse bis zum Methodenstreit, von der Spurensuche bis zum Raumverzicht. Wien. S. 9-26 (= Materialien zur Didaktik der Geographie und Wirtschaftskunde 15).

Vock, M. \& A. Gronostaj (2017): Umgang mit Heterogenität in Schule und Unterricht (1. Aufl.). FriedrichEbert-Stiftung, Berlin.

Westermann Gruppe (2020a): GEO_logisch 1. Produktinformationen. https://www.westermanngruppe.at/artikel/978-3-7034-2514-1/GEO-logisch-1 (17.12.2020)

Westermann Gruppe (2020b): Durchblick 1-4. Konzept. https://www.westermanngruppe.at/reihe/DURCHBLUNTER/Durchblick-1-4\#Konzept (17.02.2021) 